INPLASY

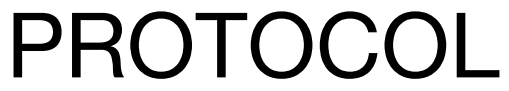

To cite: Zhao et al. A metaanalysis of colchicine in prevention of atrial fibrillation following cardiothoracic surgery or cardiac intervention. Inplasy protocol 202190004. doi:

10.37766/inplasy2021.9.0004

Received: 01 September 2021

Published: 01 September 2021

Corresponding author:

Zhao Hong

zhaohong1006@qq.com

Author Affiliation:

Chongqing Medical University, The First Affiliated Hospital of Chongqing Medical University.

Support: None.

Review Stage at time of this submission: Preliminary searches.

Conflicts of interest:

None declared.

\section{A meta-analysis of colchicine in prevention of atrial fibrillation following cardiothoracic surgery or cardiac intervention}

Zhao, $\mathrm{H}^{1}$; Chang, J2.

Review question / Objective: Question 1:Does colchicine reduce the incidence of atrial fibrillation in patients who have undergone surgery compared with the control group? Question 2:Does the dosage of colchicine affect the incidence of atrial fibrillation in patients who have undergone surgery compared with the control group? Question 3:Does the medication time of colchicine affect the incidence of atrial fibrillation in patients who have undergone surgery compared with the control group?

Information sources: We will search articles in three electronic database including PubMed, Google scholar and Cochrane Library. All the English publications until August 1st, 2021 will be searched. Reference list of all selected articles will independently screened to identify additional studies left out in the initial search.

INPLASY registration number: This protocol was registered with the International Platform of Registered Systematic Review and Meta-Analysis Protocols (INPLASY) on 01 September 2021 and was last updated on 01 September 2021 (registration number INPLASY202190004).

\section{INTRODUCTION}

Review question / Objective: Question 1:Does colchicine reduce the incidence of atrial fibrillation in patients who have undergone surgery compared with the control group? Question 2:Does the dosage of colchicine affect the incidence of atrial fibrillation in patients who have undergone surgery compared with the control group? Question 3:Does the medication time of colchicine affect the incidence of atrial fibrillation in patients who have undergone surgery compared with the control group?

Condition being studied: Colchicine, extracted from autumn crocus, is an antiinflammatory drug that has been used 
continuously for more than $\mathbf{3 0 0 0}$ years. In 1820, Pelletier and Caventou took the lead in extracting the active alkaloid from autumn crocus. It was originally used as a medicine to treat acute gouty arthritis whose adverse reactions were mainly gastrointestinal symptoms. With the continuous development of science and technology, this ancient medicine shines with new light due to its wide range of antiinflammatory activities. There is an article that summarizes the new uses of colchicine in cardiovascular disease, and it is currently believed that colchicine has potential benefits for heart disease. Atrial fibrillation is a common and extremely dangerous cardiovascular disease. Atrial fibrillation (AF) is the most common persistent arrhythmia and more than 33 million people worldwide are affected currently, and its prevalence is expected to more than double in the next $\mathbf{4 0}$ years. At present, antiarrhythmic drugs (AADs) and ablation are mainly used to prevent the occurrence of atrial fibrillation. But it cannot prevent the later recurrence of arrhythmia. And due to the insufficient efficacy of AADs, different nonantiarrhythmic therapeutic drugs, including glucocorticoid, colchicine, statins, n-3 FATTY ACIDS, have been tested. As for ablation, although it is the most effective treatment for AF, it can also cause inflammatory changes associated with early AF events, and other cardiac surgery is also associated with early AF. Colchicine has good prospects and safe results in cardiovascular patients. It may be recommended to be used in clinical trials for further study of cardiovascular diseases.

\section{METHODS}

Participant or population: Patients after cardiothoracic surgery or cardiac intervention.

Intervention: Colchicine.

Comparator: Placebo or blank control.

Study designs to be included: RCT, cohort studies.
Eligibility criteria: The inclusion criteria were as follows: 1) Randomized controlled trial with the intervention of colchicine or placebo, 2) Cohort study with the exposure factors of colchicine. 3)Patients after cardiothoracic surgery or cardiac intervention.

Information sources: We will search articles in three electronic database including PubMed, Google scholar and Cochrane Library. All the English publications until August 1st, 2021 will be searched. Reference list of all selected articles will independently screened to identify additional studies left out in the initial search.

Main outcome(s): Can Colchicine Reduce the Incidence of Atrial Fibrillation in Patients after Cardiothoracic Surgery or Cardiac Intervention. Our expected results are positive.

Quality assessment / Risk of bias analysis: We use Cochrane ROB tool to process data. Bias risk assessment items include random sequence generation (selection bias), allocation concealment (selection bias), blinding of participants and personnel (performance bias), blinding of outcome assessment (detection bias), incomplete outcome data (attrition bias), selective reporting (reporting bias), other bias.

Strategy of data synthesis: We will use "Revman" and other packages to process data. We will use random effects model or fixed effects model. $Q$ test method will be used to test heterogeneity.

Subgroup analysis: We will conduct two subgroup analyses. The first subgroup analyzes the effect of the dosage of colchicine on the incidence of atrial fibrillation, and the second subgroup analyzes the effect of medication time of colchicine on the incidence of atrial fibrillation. In the dose-effect group, the covariate is the dose of colchicine: normal dose and low-dose. In the time-effect group, the covariate is the time of colchicine use: greater than or equal to one 
month and less than one month. All patients included in the literature have undergone cardiothoracic surgery or interventional heart surgery. We will use a random effects model or a fixed effects model to analyze the heterogeneity and statistical significance of the subgroups-that is, whether colchicine is effective in preventing atrial fibrillation, and whether low-dose and short-term treatment have an effect on the efficacy of colchicine.

Sensitivity analysis: Sensitivity analysis will be done by removing one study at a time and repeating the calculations.

Country(ies) involved: China.

Keywords: colchicine, atrial fibrillation, cardiothoracic surgery, cardiac intervention.

Contributions of each author:

Author 1 - Zhao Hong.

Email: zhaohong1006@qq.com

Author 2 - Chang Jing.

Email: 1584105002@qq.com 